\title{
3D RECONSTRUCTION WITH UNCALIBRATED CAMERAS USING THE SIX-LINE CONIC VARIETY
}

\author{
Pablo Carballeira, José I. Ronda* \\ Grupo de Tratamiento de Imágenes \\ ETSI Telecomunicación \\ Univ. Politécnica de Madrid, Spain \\ $\{$ pcl, jir\}@gti.ssr.upm.es
}

Antonio Valdés

\author{
Dept. de Geometría y Topología \\ Facultad de Ciencias Matemáticas \\ Univ. Complutense de Madrid, Spain \\ valdesmoralesantonio@gmail.com
}

\begin{abstract}
We present new algorithms for the recovery of the Euclidean structure from a projective calibration of a set of cameras with square pixels but otherwise arbitrarily varying intrinsic and extrinsic parameters. Our results, based on a novel geometric approach, include a closed-form solution for the case of three cameras and two known vanishing points and an efficient one-dimensional search algorithm for the case of four cameras and one known vanishing point. In addition, an algorithm for a reliable automatic detection of vanishing points on the images is presented. These techniques fit in a 3D reconstruction scheme oriented to urban scenes reconstruction. The satisfactory performance of the techniques is demonstrated with tests on synthetic and real data.
\end{abstract}

Index Terms - 3D reconstruction, autocalibration, absolute conic, isotropic line, vanishing point, projective geometry

\section{INTRODUCTION}

The problem of obtaining a 3D reconstruction from a set of images is a central issue in modern computer vision [1]. An important practical situation is that in which relative camera positions are not available. If nothing is known about the internal parameters of the cameras then only a projective reconstruction is possible, i.e., one that differs from the actual scene in a 3D homography. However, some minimal data on the camera intrinsic parameters allow for the Euclidean upgrading of the projective reconstruction, converting it into the desired 3D scene (camera autocalibration problem)[1].

In the case of cameras with varying parameters, the usual restrictions are those associated with the pixel shape. For example, in [2] the inter-image homography is considered for the autocalibration of purely rotating cameras with varying parameters.

The characterization given in [3] and [4] of square pixel (SP) cameras has been used in [5] and [6] to calibrate cameras with varying parameters through the minimization of a cost function in terms of the projection matrices. The initialization of the algorithms were based either on a priori approximate knowledge of the internal parameters or on the assumption of constant internal parameters so that Kruppa equations can be used. In [7] the same problem is adressed through the optimization of a cost function depending on the DAQ and the intrinsic camera parameters.

*This work has been partially supported by the Spanish Administration agency CDTI under project CENIT-VISION 2007-1007, by the Ministerio de Ciencia e Innovación of the Spanish Government under project TEC2007-67764 (SmartVision), and by the Comunidad de Madrid under project S-0505/TIC-0223 (Pro-Multidis).

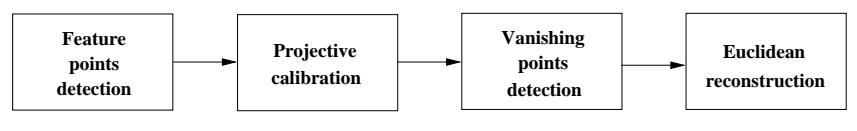

Fig. 1. Scheme of the designed 3D reconstruction system.

All the aforementioned algorithms for autocalibration of cameras with varying parameters under general motion share the limitation of needing an accurate initialization with an approximate solution.

The Absolute Quadratic Complex (AQC) [8] [9] [10], which is the mathematical object representing the set of lines intersecting the absolute conic, has been employed in a particularly successful way to obtain Euclidean upgradings in the case of cameras with square pixels. This technique is motivated from the fact that each of these cameras happens to provide two lines intersecting the absolute conic. Ten cameras provide enough equations to compute the AQC by solving an homogeneous linear system of equations.

This is an inconvenience of the AQC approach, since in general four cameras suffice to provide a discrete number of solutions for this autocalibration problem, and five cameras ensure solution uniqueness. In this work we start from the same basic geometric observation but follow a totally different approach. Instead of using the AQC, we select three of the cameras and study the set of candidate planes at infinity compatible with the restrictions imposed by these cameras. Starting from the results in [11], in which the variety of planes that intersect six lines in points of a conic is analyzed, we provide a closed-form solution for a so far (to our knowledge) unsolved problem, namely, the autocalibration of three SP cameras with varying parameters when two vanishing points (VPs) are known. As we prove, the problem has up to five posible solutions. For the case when only one VP is available we also provide a solution for four cameras using one-dimensional search. An algorithm for autocalibration without VPs in the case of five or more cameras using bidimensional search is also proposed.

We mainly consider an application environment in which the captured scene contains parallel lines, as is notably the case of urban scenes. The reconstruction scheme is shown in Figure 1. The paper is organized as follows. In Section 2 an algorithm for VP detection is presented. In Section 3 the autocalibration problem is solved using the new algorithms. Finally, experimental results are presented in Section 4. 


\section{AUTOMATIC VANISHING POINT DETECTION}

Automatic detection of VPs has been widely studied. Particularly interesting are those approaches using Hough transform techniques [12] [13] [14] [15]. In [12] a VP detection algorithm where no a priori information of the scene needed is presented. This algorithm calculates the VPs of a set of lines using a partition of the image plane and calculating the number of lines that meet the same region. This partition is built in such a way that the probability that a random line of the image meets any region is constant. In order to improve the results of this technique we have developed an optimization algorithm that minimizes the residual detection error.

In [12] lines are used as the input of the detection algorithm. In our work we use the Hough transform to obtain the initial lines from automatically detected feature points. In fact, due to the noise in the image, these points are not strictly collinear. The improvement of the adjustment of the points to their assigned lines, grouped into sets of convergent lines, is the aim of the optimization procedure.

\subsection{Vanishing point detection optimization}

Let $\mathbf{p}_{j}$ be a set of $N$ unknown 2D points aligned with the known point $\mathbf{o} \equiv\left(x_{0}, y_{0}\right)$. The maximum likelihood estimation of the common line from a set of noisy observations $\tilde{\mathbf{p}}_{j}=\left(x_{j}, y_{j}\right)$, under the hypothesis of i.i.d. isotropic gaussian noise, is the line $\mathbf{l} \equiv A x+B y+C=0$ that minimizes the sum of squared distances to the observations, and can be analytically obtained as

$$
\begin{aligned}
A & =-2 \gamma, B=\alpha-\beta+\sqrt{(\alpha-\beta)^{2}+4 \gamma^{2}}, \\
C & =-A x_{0}-B y_{0}, \\
\text { where } \alpha & =\sum_{i=1}^{N}\left(x_{i}-x_{0}\right)^{2}, \beta=\sum_{i=1}^{N}\left(y_{i}-y_{0}\right)^{2}, \\
\gamma & =\sum_{i=1}^{N}\left(x_{i}-x_{0}\right)\left(y_{i}-y_{0}\right) .
\end{aligned}
$$

We can therefore define the cost of a point $\mathbf{o}$ with respect to a set of points $\tilde{\mathbf{p}}_{j}$ as the sum of squared distances of these points to the maximum-likelihood line 1 .

In our case we have several sets of $2 \mathrm{D}$ point observations, each set corresponding to a line, and we assume that all the lines share a common point $\mathbf{o}$. Then, the maximum-likelihood estimation of $\mathbf{o}$ is given by the minimization of the sum of the costs of the point with respect to each set of points. We perform this minimization through exhaustive search in a sampled vecinity of the initial estimation.

The VP detection algorithm can be summarized as:

1. Obtain feature points and group them into lines using the Hough transform.

2. Group lines into sets of incident lines using the method described in [12]. Use as initial estimation for each intersection point (candidate VP) the centroid of the region.

3. Optimize the position of the intersection point $\mathbf{o}$ by minimizing cost in the vecinity of the initial estimation.

4. Validate candidate VPs by trying to find for each one a compatible (with respect to the projective reconstruction) candidate VP in each of the other images.

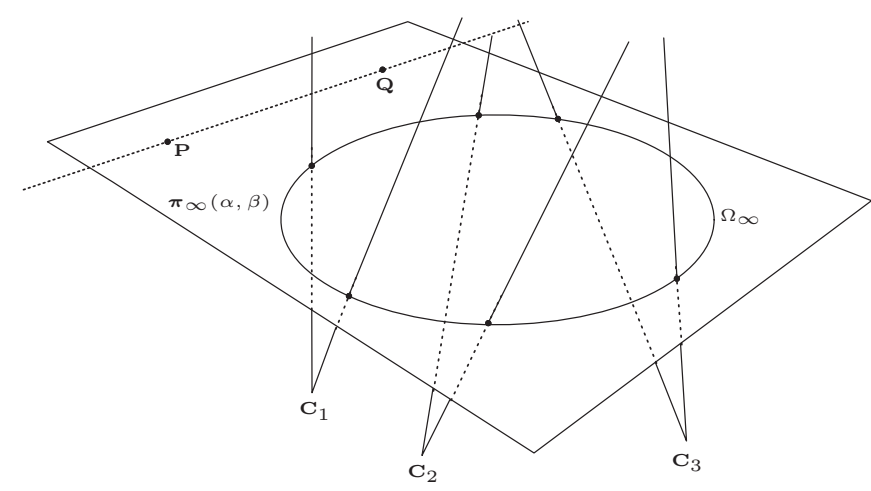

Fig. 2. Two vanishing points autocalibration scheme.

\section{AUTOCALIBRATION ALGORITHMS}

As is well known [1], a Euclidean reconstruction of the scene can be obtained if the position of the absolute conic is known.

It is easy to check [9] that the isotropic lines (backprojection of points $(1, \pm i, 0))$ of SP cameras intersect the absolute conic. Besides, in [11] an algorithm to obtain the equation of the manifold of planes that intersect six straight lines of projective three space in points of a conic section is presented. For a general configuration of lines this manifold is given by an eighth degree equation.

We can apply the results of [11] to the set of isotropic lines of three cameras. This lines are not skew, but grouped into three pairs of concurrent lines, each pair intersecting in a camera projection center (Figure 2). Therefore the variety of conic-supporting planes is not irreducible, but includes three linear components corresponding to the stars of planes through each projection center.

\subsection{Euclidean reconstruction with two vanishing points}

Points $\mathbf{P}$ and $\mathbf{Q}$ (Figure 2) are 3D points corresponding to VPs detected on the images. These two points define a pencil of planes that includes the plane at infinity. This pencil can be parameterized as

$$
\boldsymbol{\pi}(\alpha)=\boldsymbol{\pi}_{1}+\alpha \boldsymbol{\pi}_{2},
$$

where $\boldsymbol{\pi}_{1}$ and $\boldsymbol{\pi}_{2}$ are any two planes of the pencil. Using the algorithm in [11] a fifth degree polynomial equation in one variable is obtained. Therefore we have the following result.

Result 1 The Euclidean reconstruction from three views taken with cameras with square pixels and two points at infinity can be obtained by solving a quintic equation. In particular, the problem always has at least one and at most five real solutions.

\subsection{Parametrization of solutions in the case of one vanishing point}

Similarly to the case of two VPs, a star of planes, parametrized by two variables, is defined by point at infinity $\mathbf{P}$. This leads to a fifth degree polynomial equation on two variables $P(\alpha, \beta)$. A parametrization of the set of solutions can be obtained by sampling one of the variables and solving the equation for the other variable. 


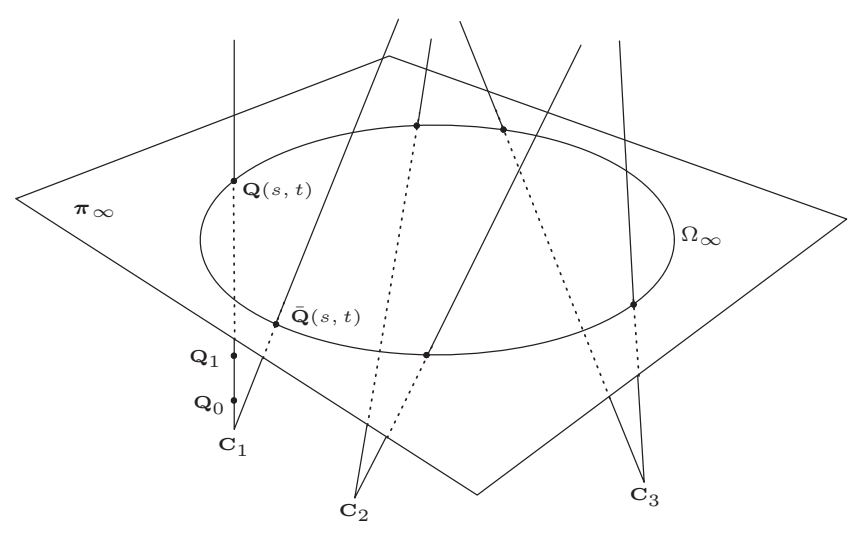

Fig. 3. A pair of points at infinity together with a candidate plane at infinite and absolute conic.

\subsection{Parametrization of solutions without vanishing points}

Now we want to obtain a parametrization of the full two-dimensional set of solutions compatible with the views from three SP cameras. This is equivalent to obtaining a parametrization of the set of real planes intersecting the six corresponding isotropic lines.

To this purpose we select one of the isotropic lines (Figure 3) and take any parametrization $\mathbf{Q}(s, t)$ of this line using two real parameters. Since we are interested in real planes, the solution plane will intersect the other isotropic line of the camera (conjugated line of the first one) in the point $\mathbf{Q}(s, t)$, and we just have to find the intersection of the variety of conic supporting planes with the pencil of planes through $\mathbf{Q}(s, t)$ and $\overline{\mathbf{Q}}(s, t)$ as in 3.1. However, as a consequence of the fact that the axis of the pencil intersects two coplanar isotropic lines, we do not find a quintic, but a second degree equation:

Result 2 The two-dimensional set of planes at infinity compatible with three views obtained with SP cameras can be parametrized by assigning to each point of one of the isotropic lines of one of the cameras the solution of a explicit second degree equation.

\section{Sketch of proof}

Using the technique of [11], Section 3, we obtain in the general case an equation of degree 12, but in our case we obtain an equation of degree 8 , as a consequence of the special configuration of the pencil. From this equation we have to remove the four spureous solutions and the two trivial solutions that appear in our case (corresponding to planes through the two optical centers of the other cameras).

\subsection{Using additional cameras}

In the case of two VPs, the selection between the five possible solutions mentioned in 3.1 can be made with the help of an additional SP camera. To this purpose we project each candidate absolute conic with the fourth camera and compute in each case the intrinsic camera parameters, being $\theta$ the skew angle and $\tau$ the aspect ratio, and selecting the one that minimizes

$$
f(\theta, \tau)=\left(1-\frac{|\theta|}{\pi / 2}\right)^{2}+(1-|\tau|)^{2} .
$$

In the case of one VP, a fourth camera ensures uniqueness of the solution in the general case. Our proposed algorithm consists in

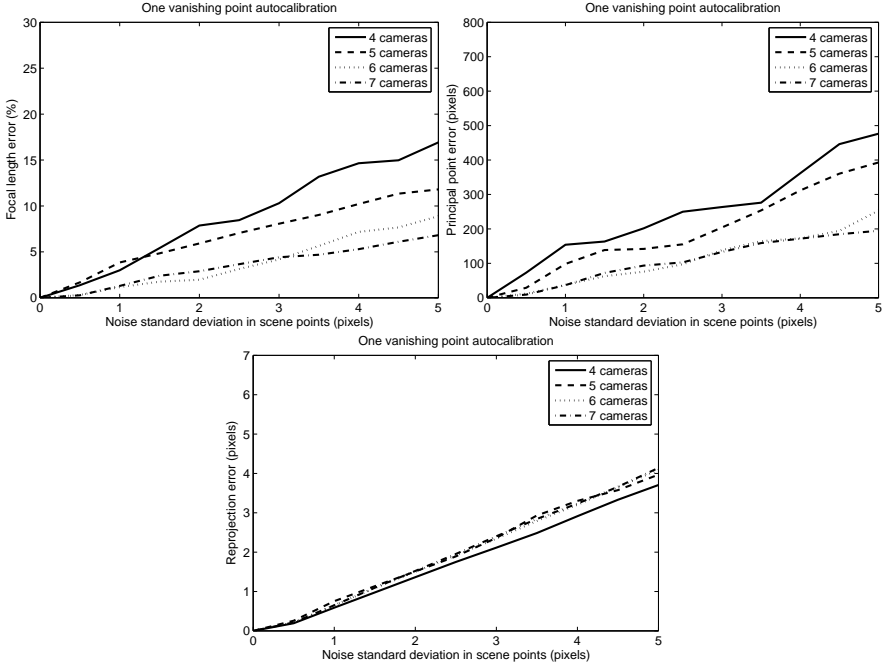

Fig. 4. Autocalibration of a synthetic scene with one vanishing point. Focal length, principal point and reprojection errors vs. noise standard deviation.

using three of the cameras and the VP to restrict the set of solutions to a curve, and to use the parametrization given in 3.2 to sample this curve, searching for the solution that minimizes (3) for the fourth camera.

A corollary of the analysis in 3.1 is that four SP cameras in general position provide a discrete number of solutions with an upper bound of $5^{3}$. If the scene has been captured with five or more SP cameras, and no VPs are available, the approach suggested by our analysis consists in using the parametrizacion of the 2D set of solutions for three of the cameras given in 3.3 to search for the solution that minimizes the sum of cost functions (3) for the remaining cameras.

\section{RESULTS WITH SYNTHETIC AND REAL DATA}

To evaluate the performance of these techniques a scenario has been simulated using a set of 100 randomly positioned 3D points captured by four to seven non-calibrated SP cameras with varying parameters.

The 3D points are placed close to the origin of coordinates and the camera arrangement intends to model a set of cameras approximately pointing towards the center of coordinates, and located all of them at a similar distance of this center. The normalized focal length is randomly selected in a distribution centered at 2000 with $10 \%$ maximum deviation. The principal point value is obtained within a uniform bidimensional distribution with support $[ \pm 160, \pm 120]$, the image size being $1600 \times 1200$.

To simulate the position of two or one VPs, intersection between known parallel lines were calculated and projected on the cameras.

Two different experiments were performed on this data set. In the first one gaussian noise was added to each coordinate of the scene points on every image. The results are presented in Figure 4.

On a second experiment noise was added to both coordinates of the VPs. The magnitude of this noise depended on the VP module to simulate reasonable VP detection error. Results are presented in Figure 5 for the case of two VPs.

To illustrate the capabilities of the proposed algorithms, including VP detection and autocalibration, a reconstruction of a typical 


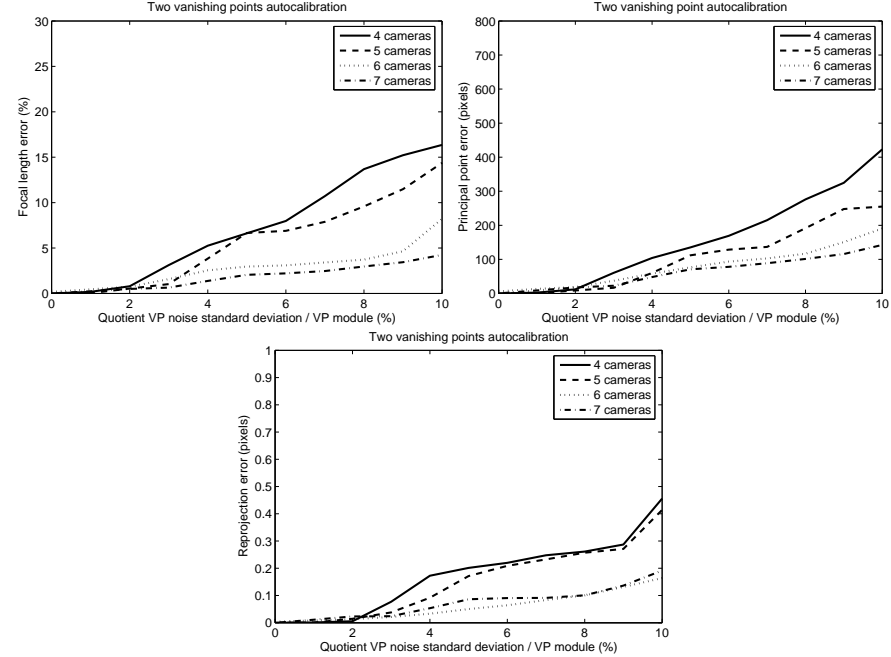

Fig. 5. Autocalibration of a synthetic scene with two vanishing points. Focal length, principal point and reprojection errors vs the standard deviation of the noise in the vanishing points.

urban scene is presented. To obtain this recostruction, the five solutions correspondig to three cameras and two VPs have been computed and two additional cameras have been used for the automatic selection of the best reconstruction. The VRML reconstruction of the scene is presented in Figure 6.

\section{CONCLUSIONS AND FUTURE WORK}

We have introduced the six-line conic variety as a tool for the Euclidean calibration of cameras with varying parameters. This approach provides solutions for the case of three cameras and two vanishing points (VPs) and parametrizations of the sets of solutions for the cases of one VP or no VP available.

The presented applications are particularly well suited to the reconstruction of scenes with parallel lines such as urban scenes.

Future work includes the practical evaluation of the technique for scenes without vanishing points, and the study of singular configurations.

\section{REFERENCES}

[1] R. Hartley and A. Zisserman, Multiple View Geometry in Computer Vision, Second Edition, Cambridge University Press, 2003.

[2] E. Hayman L. Agapito and I. Reid, "Self-calibration of rotating and zooming cameras," International Journal of Computer Vision, vol. 45, pp. 107-127, 2001.

[3] O. Faugeras, Three Dimensional Computer Vision, MIT Press, 1993.

[4] A. Heyden, Lund University, Ph.D. thesis, Geometry and algebra of multiple projective transformations, 1995.

[5] S. Bougnoux, "From projective to euclidean space under any practical situation, a criticism of self-calibration," Proc. 6th International Conference on Computer Vision, pp. 790-796, 1998.

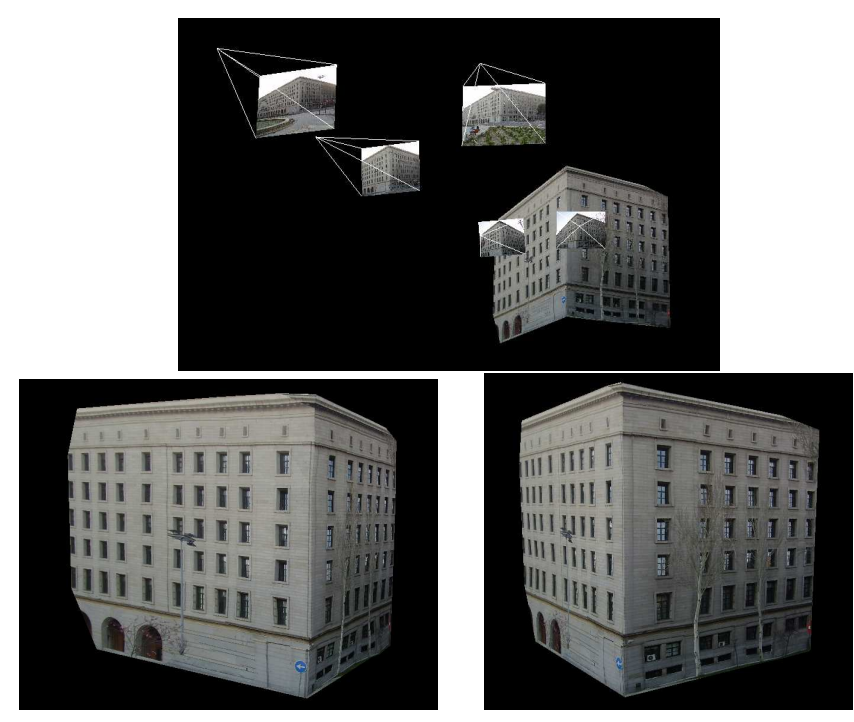

Fig. 6. VRML reconstruction of part of the Nuevos Ministerios building, (Madrid, Spain) using five uncalibrated square-pixel cameras with varying parameters and two vanishing points.

[6] A. Heyden and K. Åström, "Euclidean reconstruction from image sequences with varying and unknown focal length and principal point," Proc. IEEE Conference on Computer Vision and Pattern Recognition, 1997.

[7] M. Pollefeys, R. Koch, and L. van Gool, "Self-calibration and metric reconstruction in spite of varying and unknown internal camera parameters," International Journal of Computer Vision, vol. 1, no. 32, pp. 7-25, 1999.

[8] T. Papadopoulo M. Teillaud J. Ponce, K. McHenry and B. Triggs, "On the absolute quadratic complex and its application to autocalibration," Proc. IEEE Conference on Computer Vision and Pattern Recognition, 2005.

[9] A. Valdés and J. I. Ronda, "Camera autocalibration and the calibration pencil," Journal of Mathematical Imaging and Vision, vol. 23, no. 2, pp. 167-174, 2005.

[10] A. Valdés, J. I. Ronda, and G. Gallego, "The absolute line quadric and camera autocalibration," International Journal of Computer Vision, vol. 66, no. 3, pp. 283-303, 2006.

[11] H. P. Schröcker, "Intersection conics of six straight lines," Beitr. Algebra Geom, vol. 46, no. 2, pp. 435-446, 2005.

[12] A. Almansa, A. Desolneux, and S. Vamech, "Vanishing point detection without any a priori information," IEEE Transactions on Pattern Analysis and Machine Intelligence, vol. 25, no. 4, pp. 502-507, April 2003.

[13] R. T. Collins and R. S. Weiss, "Vanishing point calculation as a statistical inference on the unit sphere," Proc. Third International Conf. on Computer Vision, pp. 400-403, 1990.

[14] A. Minagawa, N. Tagawa, T. Moriya, and T. Goto, "Line clustering with vanishing point and vanishing line," Proc. International Conf. on Image Analysis and Processing, pp. 388-393, 1999.

[15] P. L. Palmer, M. Petrou, and J. Kittler, "Accurate line parameters from an optimising hough transform for vanishing point detection," Proc. Fourth International Conf. on Computer Vision, pp. 529-533, 1993. 